\title{
UV Written Integrated Optical Beam Combiner for Near Infrared Astronomical Interferometry
}

Svalgaard, Mikael; Olivero, Massimo; Jocou, Laurent; Berger, Jean-Philippe

Published in:

CLEO/QELS 2006 Technical Digest CD-Rom

Link to article, DOI:

10.1109/CLEO.2006.4627849

Publication date:

2006

Document Version

Publisher's PDF, also known as Version of record

Link back to DTU Orbit

Citation (APA):

Svalgaard, M., Olivero, M., Jocou, L., \& Berger, J-P. (2006). UV Written Integrated Optical Beam Combiner for Near Infrared Astronomical Interferometry. In CLEO/QELS 2006 Technical Digest CD-Rom (pp. 1-2). IEEE. https://doi.org/10.1109/CLEO.2006.4627849

\section{General rights}

Copyright and moral rights for the publications made accessible in the public portal are retained by the authors and/or other copyright owners and it is a condition of accessing publications that users recognise and abide by the legal requirements associated with these rights.

- Users may download and print one copy of any publication from the public portal for the purpose of private study or research.

- You may not further distribute the material or use it for any profit-making activity or commercial gain

- You may freely distribute the URL identifying the publication in the public portal 


\title{
UV Written Integrated Optical Beam Combiner for Near Infrared Astronomical Interferometry
}

\author{
Mikael Svalgaard \\ Research Center COM, Bldg. 345W, Technical University of Denmark, DK-2800 Lyngby, Denmark \\ svlgrd@com.dtu.dk \\ Massimo Olivero \\ PhotonLab, Politecnico di Torino, 10129 Torino, Italy \\ massimo.olivero@polito.it \\ Laurent Jocou, Jean-Philippe Berger \\ Laboratoire d'Astrophysique, Observatoire de Grenoble, F-38041 Grenoble Cedex 9, France \\ Jean-Philippe.berger@obs.ujf-grenoble.fr, jocou@obs.ujf-grenoble.fr
}

\begin{abstract}
A near infrared integrated optical beam combiner for astronomical interferometry is demonstrated for the first time by direct UV writing. High fringe contrast $>95 \%$, low total loss $(0.7 \mathrm{~dB})$, low crosstalk and broadband performance is demonstrated.

C2006 Optical Society of America

OCIS codes: (350.1260) Astronomical optics; (120.3180) Interferometry; (230.1360) Beam splitters; (130.3120) Integrated optics devices; (250.5300) Photonic integrated circuits; (160.5320) Photorefractive materials; (260.7190) Ultraviolet
\end{abstract}

\section{Introduction}

Astronomical research relies increasingly on ultra-high angular resolution optical measurements that far exceed the resolution of the Hubble Space Telescope. This can be achieved by aperture synthesis interferometry where light from several widely spaced telescopes is coherently combined and analyzed. Beam combination was initially demonstrated using bulk optical beam combiners, however as more telescopes are used for aperture synthesis this quickly becomes impractical. Recently, compact integrated optical waveguide circuits have been used to address this problem [1]. In this paper, we report for the first time, the realization of a near-infrared dual beam combiner using the technique of direct UV writing [2] where waveguides are written directly into a planar glass sample with a focused laser beam. Time-consuming and elaborate fabrication processes (e.g. reactive ion etching or ion exchange) are thus avoided and a device performance that matches or exceeds that achieved previously is demonstrated.

\section{Fabrication and design}

Channel waveguides are written into the core of a three-layer, silica-on-silicon sample using a focused $257 \mathrm{~nm}$ beam [2]. After UV writing a 3 hour annealing at $320^{\circ} \mathrm{C}$ is carried out to adjust the index step and achieve stable long term performance. The incident UV power is $45 \mathrm{~mW}$ while the scan velocity is in the range $100-900 \mu \mathrm{m} / \mathrm{s}$ depending on the desired waveguide index step and width. For most sections of the layout a scan velocity of 280 $\mu \mathrm{m} / \mathrm{s}$ is used, which yields a waveguide width of $6.1 \mu \mathrm{m}$ and a final index step of 0.0085 [3].

Optical components for astronomical interferometry must have low losses since stellar light levels are typically low (picoWatts). In addition, astronomers often wish to detect over one or more photometric bands that cover many hundreds of nanometers, which adds a requirement of broadband performance.

For this demonstration a dual beam combiner with two interferometric and two photometric outputs (Fig. 1) was fabricated. Beam interference (outputs D and E) is achieved with an ultrabroadband asymmetrical $3 \mathrm{~dB}$ directional coupler [3], while photometric outputs (outputs $\mathrm{C}$ and $\mathrm{F}$ ) are realized using $3 \mathrm{~dB}$ power splitters. The performance of these sub-components is controlled by changing the applied scan speed during UV writing. For the coupler a large bandwidth is achieved for applied scan velocities of $100,900 \mu \mathrm{m} / \mathrm{s}$ in the upper,lower arm of the central coupling region. The splitters are made symmetrical by decreasing the scan speed in one of the output arms to $200 \mu \mathrm{m} / \mathrm{s}$ to compensate for a proximity effect [4]. The total device length is $15 \mathrm{~mm}$ and the fabrication time for one component is $\sim 270$ seconds.

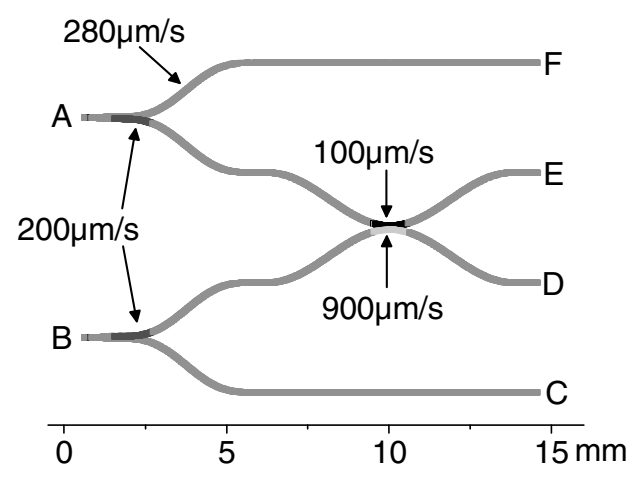

Fig. 1. Layout of UV written astronomical beam combiner. Dual beam input: A,B; interferometric output: D,E; photometric output: C,F. Scan velocities used during UV writing are indicated with arrows for various sections. 


\section{CMP3.pdf}

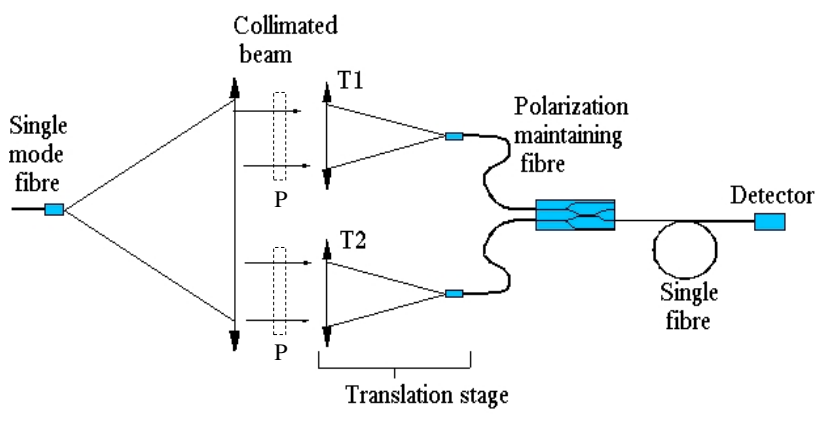

Fig. 2. Astronomical interferometric characterization test bed (T1, T2: telescope 1 and 2; P: polarizer).

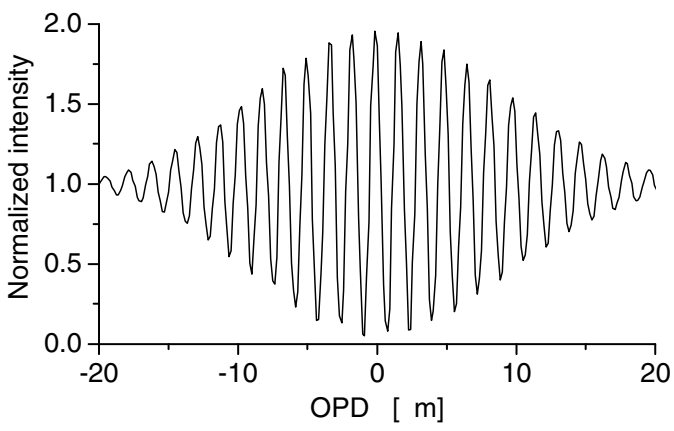

Fig. 3. Normalized interferogram measured with UV written dual beam combiner.

\section{Performance}

Optical measurements show that from 1.3-1.75 $\mu \mathrm{m}$ the total transmission loss (sum of all outputs normalized to input signal) remains flat at $\sim 0.7 \mathrm{~dB}$, while the splitting ratio is $3.2 \mathrm{~dB}$ with a ripple of $\pm 0.2 \mathrm{~dB}$. The coupling ratio exhibits a $\pm 0.5 \mathrm{~dB}$ variation from $1.5-1.75 \mu \mathrm{m}$. The actual bandwidth of the coupler is most likely larger than this since the measurement is truncated by an upper wavelength detection limit.

The beam combiner was tested on a laboratory test bed (Fig. 2) with an optical configuration similar to that used in interferometric facilities at astronomical observatories [5]. A collimated beam is produced using a single mode fiber pigtailed laser diode (1.55-1.65 $\mu \mathrm{m}$ output) and an off-axis parabolic mirror. Two telescopes (T1, T2) placed in the beam feed the integrated optical beam combiner input ports via polarization maintaining fibers. T1 is fixed while $\mathrm{T} 2$ is mounted on a translation stage to realize a controllable optical path delay (OPD). In practical systems the output ports can be read simultaneously using a CCD array, but for this work a single mode fiber coupled to a single pixel detector is used instead. Hence, real-time photometric calibration is not carried out. A polarizer is placed in front of both telescopes to improve the fringe contrast and stability by allowing the injection of linearly polarized light along the fiber+combiner fast or slow axis. A normalized interferogram is presented in Fig. 3, showing that the fringe contrast is very high, typically $95-99 \%$.

A benefit of using single mode integrated optics for beam combination is that of modal filtering, which allows for transformation of wavefront phase corrugations into power fluctuations, that can then be calibrated away using the photometric channels. This was verified by obtaining interferograms where the input fibers had deliberately been misaligned by $3 \mu \mathrm{m}$ which did not produce any measurable change in the normalized fringe contrast.

Leaky modes excited at various locations on the beam combiner (inputs, branching points, etc.) can copropagate with the main circuit and later couple to the photometric channels where they can be detected by interferometry. To test for the presence of such a problem an OPD scan is performed while both inputs are fed and the signal on a photometric channel is detected. There are no visible fringes on the direct signal, however a power spectral density plot reveals a weak peak at the interferometric fringe frequency and a cross-talk level of $\sim 40 \mathrm{~dB}$, which is excellent for astronomical measurements.

\section{Conclusion}

An integrated optical beam combiner for near infrared astronomical interferometry has been realized by the UV writing technique. The total transmission loss is $\sim 0.7 \mathrm{~dB}$ and a fringe contrast of $95-99 \%$ was measured in a dual telescope test bed. The device yields a very high fringe contrast over a wide wavelength range, while providing low cross talk between interferometric and photometric output channels. The demonstrated device performance matches or exceeds that obtained previously with other, more elaborate waveguide fabrication techniques.

\section{References}

[1] F. Malbet et al., "Integrated optics for astronomical interferometry. I. Concept and astronomical applications,” Astr. Astroph. Suppl. Ser. 138, $135-145,1999$

[2] M. Svalgaard et al., "Direct UV-writing of buried single-mode channel waveguides in Ge-doped silica films," Elec. Lett. 30, 1401-1402, 1994

[3] M. Olivero et al., "Direct UV-written Broadband Directional Planar Waveguide Couplers," Opt. Expr. 13, 2005

[4] K. Færch et al., "Symmetrical waveguide device fabricated by direct UV writing," IEEE Photon. Technol. Lett. 14, 173-175, 2002

[5] J.-P. Berger et al.., ’'Integrated optics for astronomical interferometry. IV. First measurements of stars,” Astr. \& Astroph. 376, L31-L34, 2001 